\title{
An economical Nanopore sequencing assay for human papillomavirus (HPV) genotyping
}

Wai Sing Chan, Tsun Leung Chan, Chun Hang Au, Chin Pang Leung, Man Yan To, Man Kin Ng, Sau Man Leung, May Kwok Mei Chan, Edmond Shiu Kwan Ma and Bone Siu Fai Tang ${ }^{*}$

\begin{abstract}
Background: Human papillomavirus (HPV) testing has been employed by several European countries to augment cytology-based cervical screening programs. A number of research groups have demonstrated potential utility of next-generation sequencing (NGS) for HPV genotyping, with comparable performance and broader detection spectrum than current gold standards. Nevertheless, most of these NGS platforms may not be the best choice for medium sample throughput and laboratories with less resources and space. In light of this, we developed a Nanopore sequencing assay for HPV genotyping and compared its performance with cobas HPV Test and Roche Linear Array HPV Genotyping Test (LA).
\end{abstract}

Methods: Two hundred and one cervicovaginal swabs were routinely tested for Papanicolaou smear, cobas HPV Test and LA. Residual DNA was used for Nanopore protocol after routine testing. Briefly, HPV L1 region was amplified using PGMY and MGP primers, and PCR-positive specimens were sequenced on MinION flow cells (R9.4.1). Data generated in first $2 \mathrm{~h}$ were aligned with reference sequences from Papillomavirus Episteme database for genotyping.

Results: Nanopore detected 96 HPV-positive (47.76\%) and 95 HPV-negative (47.26\%) specimens, with 10 lacking $\beta$ globin band and not further analyzed (4.98\%). Substantial agreement was achieved with cobas HPV Test and LA (K: 0.83-0.93). In particular, Nanopore appeared to be more sensitive than cobas HPV Test for HPV $52(n=7)$. For LA, Nanopore revealed higher concordance for high-risk ( $\mathrm{k}: 0.93$ ) than non-high risk types ( $\mathrm{k}: 0.83$ ), and with similar high-risk positivity in each cytology grading. Nanopore also provided better resolution for HPV 52 in 3 specimens co-infected with HPV 33 or 58, and for HPV 87 which was identified as HPV 84 by LA. Interestingly, Nanopore identified 5 additional HPV types, with an unexpected high incidence of HPV $90(n=12)$ which was reported in North America and Belgium but not in Hong Kong.

Conclusions: We developed a Nanopore workflow for HPV genotyping which was economical (about USD 50.77 per patient specimen for 24-plex runs), and with comparable or better performance than 2 reference methods in the market. Future prospective study with larger sample size is warranted to further evaluate test performance and streamline the protocol.

Keywords: Cervical cancer, HPV, Nanopore, NGS

\footnotetext{
* Correspondence: bsftang@gmail.com

Department of Pathology, Hong Kong Sanatorium \& Hospital, Hong Kong, China
} 


\section{Introduction}

Human papillomavirus (HPV) is generally accepted as the causative agent of cervical cancer (CC) [1], which was first unmasked by the landmark studies of Meisels and Fortin [2] and Purola and Savia [3]. Currently, there are 198 reference HPV types listed on Papillomavirus Episteme (PaVE) database, and at least 12 were classified as high-risk by World Health Organization (WHO) International Agency for Research on Cancer (IARC) Monographs Working Group [4-6]. HPV testing has been adopted by several European countries for primary $\mathrm{CC}$ screening, to augment cytology-based screening programs [7, 8]. A number of HPV assays are available commercially, which are mainly based on direct HPV genome detection, HPV DNA amplification and E6/ E7 mRNA detection [9]. Recent advent of next-generation sequencing (NGS) technologies has facilitated high throughput tools for infectious disease diagnostics and epidemiological research. Several research groups have explored utility of Illumina MiSeq and Ion Torrent platforms for HPV genotyping, with comparable sensitivity to well-established line blot assays and broader detection spectrum [10-12]. While the reagent cost is comparable to existing commercial assays for large sample batches, these NGS platforms may not be the best choice for medium sample throughput and laboratories with less resources and space. In this regard, portable Nanopore sequencers may allow more flexibility with shorter sequencing time and lower reagent cost. In light of this, we developed a Nanopore HPV genotyping protocol using 2 published primer sets, and compared its performance with 2 commercial HPV assays: cobas HPV Test and Roche Linear Array HPV Genotyping Test (LA).

\section{Methods}

\section{Specimens}

Two hundred and one cervicovaginal swabs were collected from March to July, 2019 in Hong Kong Sanatorium \& Hospital. The swabs were preserved in SurePath preservative fluid (Becton, Dickson and Company, Sparks, MD, USA) and routinely tested for Papanicolaou smear (Pap smear, following The Bethesda System for reporting), cobas HPV Test and LA (Roche Diagnostics, Mannheim, Germany). Routine test results are shown in Table 1.

\section{DNA extraction}

DNA extraction and cobas HPV Test were performed using cobas 4800 system (Roche Diagnostics, Rotkreuz, Switzerland). Briefly, $500 \mu \mathrm{L}$ of cervicovaginal specimen was added to $500 \mu \mathrm{L}$ of sample preparation buffer and heated at $120^{\circ} \mathrm{C}$ for $20 \mathrm{~min}$. The mixture was brought to ambient temperature for $10 \mathrm{~min}$ and processed on cobas $\times 480$ using 'high-risk HPV DNA PCR' protocol. Realtime polymerase chain reaction (PCR) was performed on cobas z 480. Fifty microliter of DNA extract was used for LA according to manufacturer's recommendations. Residual DNA was used for Nanopore protocol after routine testing.

\section{HPV PCR}

For each specimen, L1 region of HPV genome was amplified in 2 separate PCRs using PGMY and MGP primer sets $[13,14]$. Primer sequences and cycling conditions are shown in Tables 2 and 3. Human $\beta$-globin gene was used as inhibition control and contamination was monitored by negative extraction control. Five microliter of each PCR amplicon was electrophoresized in $2 \%$ agarose gel (Invitrogen, Carlsbad, CA, USA) and analyzed. PCRpositive specimens were sequenced using Nanopore MinION.

\section{Nanopore sequencing library preparation}

PGMY and MGP PCR amplicons of each positive specimen were pooled and purified using AMPure XP beads (Beckman-Coulter, Brea, CA, USA). Nanopore sequencing libraries were prepared from purified amplicons using Ligation Sequencing Kit 1D (SQK-LSK109) and PCR-free Native Barcoding Expansion Kit (EXP-NBD104/114) (Oxford Nanopore Technologies, Oxford, England). The barcoded libraries were loaded and sequenced on MinION flow cells (FLOMIN106D R9.4.1, Oxford Nanopore Technologies, Oxford, England) after quality control runs.

\section{Data analysis}

Data from first $2 \mathrm{~h}$ of sequencing runs was analyzed. FASTQ files generated by live basecalling (MinKNOW version 2.0) were demultiplexed using 'FASTQ Barcoding' workflow on EPI2ME (Oxford Nanopore Technologies, Oxford, England) with default minimum qscore of 7, 'auto' and 'split by barcode' options. FASTQ files of each specimen were concatenated into a single file and analyzed using a 2-step custom workflow on Galaxy bioinformatics platform. Briefly, FASTQ files were converted into FASTA format, followed by aligning sequences against HPV reference genomes from PaVE database using NCBI BLAST+ blastn (Galaxy version 1.1.1). PGMY and MGP reads were sorted based on sequence length and analyzed individually. Threshold of each run was derived from average number of background reads plus 10 standard deviations, which were calculated using interquartile rule, excluding first and last quartiles. A positive HPV call was based on either (1) the number of reads for a particular HPV type was above threshold, or (2) the specimen had the highest number of reads for a particular HPV type. All positive calls were further assessed by aligning FASTQ sequences 
Table 1 Results of Pap smear, cobas HPV Test, Roche Linear Array HPV Genotyping Test, and Nanopore sequencing

\begin{tabular}{|c|c|c|c|c|c|c|c|c|c|}
\hline \multirow[t]{2}{*}{ Patient } & \multirow[t]{2}{*}{ Pap smear } & \multicolumn{2}{|c|}{ Roche Linear Array } & \multirow[t]{2}{*}{ Cobas HPV } & \multicolumn{2}{|c|}{ Nanopore (PGMY) } & \multicolumn{2}{|c|}{ Nanopore (MGP) } & \multirow{2}{*}{$\begin{array}{l}\text { Total } \\
\text { HPV } \\
\text { reads }\end{array}$} \\
\hline & & $\mathrm{HR}$ & Non-HR & & $\mathrm{HR}$ & Non-HR & $\mathrm{HR}$ & Non-HR & \\
\hline 1 & AGUS & Neg & Neg & Neg & Neg & Neg & Neg & Neg & ND \\
\hline 2 & $\mathrm{ASCH}$ & 52,59 & 62 & Other HR & 59 & Neg & 59 & 90 & 4956 \\
\hline 3 & ASCUS & 52 & 55 & Neg & 52 & 55 & Neg & Neg & 4262 \\
\hline 4 & ASCUS & Neg & Neg & Neg & Neg & Neg & Neg & Neg & ND \\
\hline 5 & ASCUS & 31,33 & 54 & Other HR & $31,33,52$ & Neg & Neg & 90 & 8973 \\
\hline 6 & ASCUS & Neg & Neg & Neg & Neg & Neg & Neg & Neg & ND \\
\hline 7 & ASCUS & 31 & Neg & Other HR & Neg & Neg & 31 & Neg & 1430 \\
\hline 8 & ASCUS & Neg & Neg & Neg & Neg & Neg & Neg & Neg & ND \\
\hline 9 & ASCUS & Neg & 81 & Neg & Neg & 81 & Neg & 81 & 48,477 \\
\hline 10 & ASCUS & 18 & Neg & 18 & 18 & Neg & 18 & Neg & 16,206 \\
\hline 11 & ASCUS & Neg & Neg & Neg & Neg & Neg & Neg & Neg & ND \\
\hline 12 & ASCUS & Neg & Neg & Neg & Neg & Neg & Neg & Neg & ND \\
\hline 13 & ASCUS & 52 & 53,54 & Other HR & 52 & $44,53,74$ & 52 & 74,90 & 15,419 \\
\hline 14 & ASCUS & Neg & Neg & Neg & Neg & Neg & Neg & Neg & ND \\
\hline 15 & ASCUS & Neg & Neg & Neg & Neg & Neg & Neg & Neg & ND \\
\hline 16 & ASCUS & 52 & 81 & Neg & 52 & 81 & Neg & 81 & 8873 \\
\hline 17 & ASCUS & 52 & 54 & Other HR & 52 & 54 & 52 & 54 & 36,258 \\
\hline 18 & ASCUS & 52,59 & 11 & Other HR & 52,59 & 11 & 52,59 & 11 & 44,702 \\
\hline 19 & ASCUS & Neg & Neg & Neg & \multicolumn{2}{|c|}{ PCR inhibition } & & & \\
\hline 20 & ASCUS & Neg & Neg & Neg & Neg & Neg & Neg & $\mathrm{Neg}$ & 7 \\
\hline 21 & ASCUS & Neg & Neg & Neg & Neg & Neg & Neg & Neg & ND \\
\hline 22 & ASCUS & Neg & Neg & Neg & Neg & Neg & Neg & Neg & ND \\
\hline 23 & ASCUS & 39 & 61,72 & Other HR & 39 & 61,72 & 39 & 87 & 1624 \\
\hline 24 & ASCUS & 66 & Neg & Other HR & 66 & Neg & 66 & Neg & 10,383 \\
\hline 25 & ASCUS & 68 & 61 & Other HR & Neg & 61 & Neg & 61 & 10,644 \\
\hline 26 & ASCUS & Neg & Neg & Neg & Neg & Neg & Neg & 90 & 541 \\
\hline 27 & ASCUS & 52 & Neg & Neg & 52 & Neg & Neg & 87 & 3614 \\
\hline 28 & ASCUS & Neg & 62 & Neg & Neg & 62 & Neg & 62 & 45 \\
\hline 29 & ASCUS & Neg & Neg & Neg & Neg & $\mathrm{Neg}$ & Neg & $\mathrm{Neg}$ & ND \\
\hline 30 & ASCUS & 35 & Neg & Other HR & 35 & Neg & 35 & Neg & 1641 \\
\hline 31 & ASCUS & Neg & Neg & Neg & Neg & Neg & Neg & Neg & ND \\
\hline 32 & ASCUS & 52 & Neg & Other HR & 52 & Neg & 52 & Neg & 399 \\
\hline 33 & ASCUS & Neg & Neg & Neg & Neg & Neg & Neg & Neg & ND \\
\hline 34 & ASCUS & 51 & 84 & Other HR & 51 & Neg & Neg & Neg & 1853 \\
\hline 35 & ASCUS & Neg & Neg & Neg & Neg & 74 & Neg & 74 & 11,499 \\
\hline 36 & ASCUS & Neg & Neg & Neg & Neg & Neg & Neg & Neg & 93 \\
\hline 37 & ASCUS & 51 & Neg & Other HR & 51 & Neg & 51 & Neg & 2897 \\
\hline 38 & ASCUS & Neg & $40,55,83$ & Neg & Neg & $40,55,83$ & Neg & $40,55,83$ & 47,736 \\
\hline 39 & ASCUS & Neg & Neg & Neg & Neg & Neg & Neg & Neg & ND \\
\hline 40 & ASCUS & 58 & $53,55,62$ & Other HR & 52,58 & $53,55,62,74$ & 52 & $53,62,74$ & 42,106 \\
\hline 41 & ASCUS & 52 & 42,73 & Other HR & 52 & 42,73 & 52 & 42,73 & 15,778 \\
\hline 42 & ASCUS & Neg & Neg & Neg & Neg & Neg & Neg & Neg & 116 \\
\hline 43 & HSIL & 16 & Neg & 16 & 16 & Neg & 16 & Neg & 15,918 \\
\hline
\end{tabular}


Table 1 Results of Pap smear, cobas HPV Test, Roche Linear Array HPV Genotyping Test, and Nanopore sequencing (Continued)

\begin{tabular}{|c|c|c|c|c|c|c|c|c|c|}
\hline \multirow[t]{2}{*}{ Patient } & \multirow[t]{2}{*}{ Pap smear } & \multicolumn{2}{|c|}{ Roche Linear Array } & \multirow[t]{2}{*}{ Cobas HPV } & \multicolumn{2}{|c|}{ Nanopore (PGMY) } & \multicolumn{2}{|c|}{ Nanopore (MGP) } & \multirow{2}{*}{$\begin{array}{l}\text { Total } \\
\text { HPV } \\
\text { reads }\end{array}$} \\
\hline & & $H R$ & Non-HR & & $\mathrm{HR}$ & Non-HR & $\mathrm{HR}$ & Non-HR & \\
\hline 44 & HSIL & 16 & Neg & 16 & 16 & Neg & 16 & Neg & 34,654 \\
\hline 45 & HSIL & 59 & Neg & Other HR & 59 & Neg & 59 & Neg & 15,381 \\
\hline 46 & HSIL & 31,58 & Neg & Other HR & 31,58 & Neg & 31,58 & Neg & 3367 \\
\hline 47 & LSIL & 52,68 & 84 & Other HR & 52,68 & 84 & 52,68 & 84,90 & 24,366 \\
\hline 48 & LSIL & 66 & 84 & Other HR & 66 & 44,84 & 66 & 44 & 57,206 \\
\hline 49 & LSIL & 52 & Neg & Neg & 52 & Neg & 52 & Neg & 14,516 \\
\hline 50 & LSIL & Neg & 40,53 & Neg & Neg & 40,53 & Neg & 40,53 & 9265 \\
\hline 51 & LSIL & 52 & 11,81 & Other HR & 52 & 11,81 & 52 & $11,43,81$ & 29,748 \\
\hline 52 & LSIL & 66 & Neg & Other HR & 66 & Neg & 66 & Neg & 40,328 \\
\hline 53 & LSIL & 51 & Neg & Other HR & 51 & Neg & 51 & 43,90 & 4454 \\
\hline 54 & LSIL & $16,51,56$ & $54,62,81$ & 16, other HR & $16,51,56$ & $54,62,81$ & 16,51 & $40,62,81$ & 20,455 \\
\hline 55 & LSIL & 56 & 53 & Other HR & 56 & 53 & 56 & 53 & 28,377 \\
\hline 56 & LSIL & Neg & $\mathrm{Neg}$ & Neg & Neg & Neg & Neg & Neg & ND \\
\hline 57 & LSIL & 66 & $54,55,81$ & Other HR & 66 & $54,55,81$ & 66 & $55,81,90$ & 25,606 \\
\hline 58 & LSIL & 52 & $\mathrm{Neg}$ & Neg & 52 & 42 & 52 & 90 & 15,103 \\
\hline 59 & LSIL & 59 & Neg & Other HR & 59 & Neg & Neg & Neg & 11,235 \\
\hline 60 & LSIL & 59 & 89 & Neg & 59 & 89 & Neg & 89 & 67,220 \\
\hline 61 & LSIL & 56 & 82 & Other HR & 56 & 82 & 56 & 43,82 & 42,160 \\
\hline 62 & LSIL & 52 & Neg & Other HR & 52 & Neg & 52 & Neg & 39,323 \\
\hline 63 & LSIL & 33,51 & Neg & Other HR & 33,51 & 44 & 51 & 44 & 19,704 \\
\hline 64 & $\mathrm{LSIL}+\mathrm{ASCH}$ & 51 & Neg & Other HR & 51 & Neg & 51 & Neg & 4621 \\
\hline 65 & NIL & 16 & Neg & 16 & 16 & Neg & 16 & Neg & 1958 \\
\hline 66 & NIL & Neg & Neg & Neg & Neg & Neg & Neg & Neg & ND \\
\hline 67 & NIL & Neg & $\mathrm{Neg}$ & Neg & Neg & Neg & Neg & Neg & ND \\
\hline 68 & NIL & Neg & Neg & Neg & 59 & Neg & 59 & Neg & 2455 \\
\hline 69 & NIL & Neg & Neg & Neg & Neg & 87 & Neg & 87 & 8775 \\
\hline 70 & NIL & Neg & Neg & Neg & Neg & Neg & Neg & Neg & ND \\
\hline 71 & NIL & Neg & Neg & Neg & Neg & Neg & Neg & Neg & ND \\
\hline 72 & NIL & Neg & $\mathrm{Neg}$ & Neg & Neg & Neg & Neg & Neg & ND \\
\hline 73 & NIL & Neg & Neg & Neg & Neg & Neg & Neg & Neg & ND \\
\hline 74 & NIL & 58 & Neg & Other HR & 58 & Neg & 52,58 & 62 & 8619 \\
\hline 75 & NIL & 58 & Neg & Other HR & 58 & Neg & 58 & Neg & 13,149 \\
\hline 76 & NIL & Neg & Neg & Neg & Neg & Neg & Neg & Neg & ND \\
\hline 77 & NIL & Neg & $\mathrm{Neg}$ & Neg & Neg & Neg & Neg & Neg & ND \\
\hline 78 & NIL & Neg & Neg & Neg & Neg & Neg & Neg & 90 & 2289 \\
\hline 79 & NIL & 56 & 70 & Other HR & Neg & 44,70 & 56 & 44,70 & 7855 \\
\hline 80 & NIL & Neg & Neg & Neg & PCR inhib & & & & \\
\hline 81 & NIL & Neg & Neg & Neg & Neg & Neg & Neg & Neg & 74 \\
\hline 82 & NIL & Neg & 42 & Neg & Neg & Neg & Neg & 42 & 1406 \\
\hline 83 & NIL & Neg & Neg & Neg & Neg & 74 & Neg & 74 & 7441 \\
\hline 84 & NIL & Neg & Neg & Neg & Neg & Neg & Neg & Neg & ND \\
\hline 85 & NIL & Neg & 82 & Neg & Neg & 82 & Neg & 82 & 1162 \\
\hline 86 & NIL & Neg & 62 & $\mathrm{Neg}$ & Neg & 62 & Neg & 62 & 65,368 \\
\hline
\end{tabular}


Table 1 Results of Pap smear, cobas HPV Test, Roche Linear Array HPV Genotyping Test, and Nanopore sequencing (Continued)

\begin{tabular}{|c|c|c|c|c|c|c|c|c|c|}
\hline \multirow[t]{2}{*}{ Patient } & \multirow[t]{2}{*}{ Pap smear } & \multicolumn{2}{|c|}{ Roche Linear Array } & \multirow[t]{2}{*}{ Cobas HPV } & \multicolumn{2}{|c|}{ Nanopore (PGMY) } & \multicolumn{2}{|c|}{ Nanopore (MGP) } & \multirow{2}{*}{$\begin{array}{l}\text { Total } \\
\text { HPV } \\
\text { reads }\end{array}$} \\
\hline & & $\mathrm{HR}$ & Non-HR & & $\mathrm{HR}$ & Non-HR & $\mathrm{HR}$ & Non-HR & \\
\hline 87 & NIL & Neg & Neg & Neg & Neg & Neg & Neg & Neg & ND \\
\hline 88 & NIL & Neg & Neg & Neg & Neg & Neg & Neg & Neg & ND \\
\hline 89 & NIL & Neg & Neg & Neg & Neg & Neg & Neg & Neg & ND \\
\hline 90 & NIL & Neg & Neg & Neg & Neg & Neg & Neg & Neg & 142 \\
\hline 91 & NIL & 39,52 & Neg & Other HR & 52 & Neg & 52 & 90 & 15,703 \\
\hline 92 & NIL & 68 & Neg & Other HR & 68 & 42 & 68 & Neg & 19,777 \\
\hline 93 & NIL & Neg & Neg & Neg & Neg & Neg & Neg & Neg & ND \\
\hline 94 & NIL & Neg & Neg & Neg & Neg & Neg & Neg & Neg & ND \\
\hline 95 & NIL & Neg & Neg & Neg & Neg & Neg & Neg & Neg & ND \\
\hline 96 & NIL & 52 & Neg & Neg & 52 & Neg & 52 & Neg & 5242 \\
\hline 97 & NIL & Neg & Neg & Neg & Neg & Neg & Neg & Neg & ND \\
\hline 98 & NIL & Neg & $\mathrm{Neg}$ & Neg & Neg & Neg & $\mathrm{Neg}$ & Neg & ND \\
\hline 99 & NIL & Neg & $\mathrm{Neg}$ & Neg & Neg & Neg & Neg & Neg & 41 \\
\hline 100 & NIL & 52 & Neg & Other HR & 52 & Neg & 52 & Neg & 24,478 \\
\hline 101 & NIL & Neg & 61 & Neg & \multicolumn{2}{|c|}{ PCR inhibition } & & & \\
\hline 102 & NIL & Neg & Neg & Neg & Neg & Neg & Neg & Neg & 72 \\
\hline 103 & NIL & 39 & Neg & Neg & Neg & Neg & $\mathrm{Neg}$ & Neg & ND \\
\hline 104 & NIL & Neg & 62,84 & Neg & Neg & 62 & Neg & 62 & 3589 \\
\hline 105 & NIL & Neg & 71 & $\mathrm{Neg}$ & Neg & Neg & Neg & Neg & ND \\
\hline 106 & NIL & Neg & Neg & Neg & Neg & Neg & Neg & Neg & ND \\
\hline 107 & NIL & 52 & 62 & Other HR & 52 & $44,53,62$ & 52 & 44 & 18,086 \\
\hline 108 & NIL & Neg & Neg & Neg & Neg & Neg & Neg & Neg & ND \\
\hline 109 & NIL & Neg & Neg & Neg & Neg & Neg & Neg & Neg & ND \\
\hline 110 & NIL & Neg & Neg & Neg & Neg & Neg & $\mathrm{Neg}$ & Neg & ND \\
\hline 111 & NIL & Neg & 84 & Neg & Neg & Neg & $\mathrm{Neg}$ & Neg & ND \\
\hline 112 & NIL & 16,52 & Neg & 16 & 16,52 & Neg & 16 & Neg & 72,357 \\
\hline 113 & NIL & Neg & Neg & Neg & Neg & Neg & Neg & Neg & ND \\
\hline 114 & NIL & Neg & 55,89 & Neg & Neg & $26,55,89$ & 59 & $26,55,62,89$ & 8926 \\
\hline 115 & NIL & Neg & $\mathrm{Neg}$ & Neg & Neg & Neg & $\mathrm{Neg}$ & 74 & 1586 \\
\hline 116 & NIL & Neg & 81 & Neg & Neg & Neg & Neg & Neg & ND \\
\hline 117 & NIL & Neg & $\mathrm{Neg}$ & Neg & Neg & Neg & Neg & Neg & ND \\
\hline 118 & NIL & Neg & 6,62 & Neg & Neg & 6,62 & Neg & 6,62 & 9414 \\
\hline 119 & NIL & Neg & Neg & Neg & Neg & Neg & Neg & Neg & ND \\
\hline 120 & NIL & Neg & 54 & Neg & Neg & Neg & Neg & Neg & ND \\
\hline 121 & NIL & Neg & Neg & Neg & \multicolumn{2}{|c|}{ PCR inhibition } & & & \\
\hline 122 & NIL & Neg & Neg & Neg & Neg & Neg & Neg & Neg & 8 \\
\hline 123 & NIL & 68 & Neg & Other HR & Neg & Neg & $\mathrm{Neg}$ & Neg & ND \\
\hline 124 & NIL & Neg & 81 & Neg & Neg & 81 & $\mathrm{Neg}$ & 81 & 8735 \\
\hline 125 & NIL & Neg & 84 & Neg & Neg & Neg & Neg & 87 & 1025 \\
\hline 126 & NIL & Neg & Neg & Neg & Neg & Neg & Neg & 90 & 1719 \\
\hline 127 & NIL & Neg & Neg & Neg & Neg & Neg & Neg & Neg & ND \\
\hline 128 & NIL & Neg & Neg & Neg & Neg & Neg & Neg & Neg & ND \\
\hline 129 & NIL & Neg & Neg & Neg & Neg & Neg & Neg & Neg & 10 \\
\hline
\end{tabular}


Table 1 Results of Pap smear, cobas HPV Test, Roche Linear Array HPV Genotyping Test, and Nanopore sequencing (Continued)

\begin{tabular}{|c|c|c|c|c|c|c|c|c|c|}
\hline \multirow[t]{2}{*}{ Patient } & \multirow[t]{2}{*}{ Pap smear } & \multicolumn{2}{|c|}{ Roche Linear Array } & \multirow[t]{2}{*}{ Cobas HPV } & \multicolumn{2}{|c|}{ Nanopore (PGMY) } & \multicolumn{2}{|c|}{ Nanopore (MGP) } & \multirow{2}{*}{$\begin{array}{l}\text { Total } \\
\text { HPV } \\
\text { reads }\end{array}$} \\
\hline & & $\mathrm{HR}$ & Non-HR & & $\mathrm{HR}$ & Non-HR & $\mathrm{HR}$ & Non-HR & \\
\hline 130 & NIL & Neg & Neg & Neg & Neg & Neg & Neg & Neg & ND \\
\hline 131 & NIL & Neg & 84 & Neg & Neg & Neg & Neg & Neg & ND \\
\hline 132 & NIL & Neg & Neg & Neg & Neg & Neg & Neg & Neg & ND \\
\hline 133 & NIL & 59 & 62,71 & Other HR & Neg & Neg & Neg & Neg & 30 \\
\hline 134 & NIL & Neg & Neg & Neg & Neg & Neg & $\mathrm{Neg}$ & Neg & ND \\
\hline 135 & NIL & Neg & Neg & Neg & Neg & Neg & $\mathrm{Neg}$ & Neg & 522 \\
\hline 136 & NIL & Neg & Neg & Neg & Neg & Neg & Neg & Neg & ND \\
\hline 137 & NIL & 51 & 84 & Other HR & \multicolumn{2}{|c|}{ PCR inhibition } & & & \\
\hline 138 & NIL & 39 & Neg & Other HR & 39 & Neg & 39 & Neg & 19,305 \\
\hline 139 & NIL & Neg & Neg & Neg & Neg & Neg & Neg & Neg & 195 \\
\hline 140 & NIL & Neg & Neg & Neg & Neg & Neg & Neg & Neg & ND \\
\hline 141 & NIL & Neg & Neg & Neg & Neg & Neg & $\mathrm{Neg}$ & Neg & 23 \\
\hline 142 & NIL & Neg & Neg & Neg & Neg & Neg & $\mathrm{Neg}$ & Neg & ND \\
\hline 143 & NIL & Neg & 42,81 & Neg & Neg & $40,74,81$ & Neg & $40,74,81,87$ & 19,118 \\
\hline 144 & NIL & Neg & $\mathrm{Neg}$ & Neg & Neg & Neg & Neg & Neg & ND \\
\hline 145 & NIL & Neg & Neg & Neg & Neg & Neg & Neg & Neg & ND \\
\hline 146 & NIL & Neg & $\mathrm{Neg}$ & Neg & Neg & Neg & $\mathrm{Neg}$ & Neg & ND \\
\hline 147 & NIL & Neg & Neg & Neg & Neg & Neg & Neg & Neg & 40 \\
\hline 148 & NIL & 59 & Neg & Neg & 59 & Neg & Neg & Neg & 12,681 \\
\hline 149 & NIL & Neg & Neg & Neg & Neg & Neg & Neg & Neg & 14 \\
\hline 150 & NIL & Neg & Neg & Neg & \multicolumn{2}{|c|}{ PCR inhibition } & & & \\
\hline 151 & NIL & Neg & Neg & Neg & Neg & Neg & Neg & Neg & 79 \\
\hline 152 & NIL & Neg & 62 & Neg & Neg & 62 & Neg & 62 & 14,353 \\
\hline 153 & NIL & Neg & Neg & Neg & Neg & Neg & $\mathrm{Neg}$ & Neg & ND \\
\hline 154 & NIL & Neg & Neg & Neg & Neg & Neg & $\mathrm{Neg}$ & Neg & ND \\
\hline 155 & NIL & Neg & Neg & Neg & Neg & Neg & Neg & Neg & ND \\
\hline 156 & NIL & 52 & 54 & Neg & 52 & 54 & 52 & 54 & 18,397 \\
\hline 157 & NIL & 39,52 & 53,61 & Other HR & 39 & 53,61 & 39 & 53,61 & 20,332 \\
\hline 158 & NIL & Neg & $\mathrm{Neg}$ & Neg & Neg & Neg & $\mathrm{Neg}$ & Neg & ND \\
\hline 159 & NIL & Neg & Neg & Neg & Neg & Neg & Neg & Neg & 60 \\
\hline 160 & NIL & Neg & Neg & Neg & \multicolumn{2}{|c|}{ PCR inhibition } & & & \\
\hline 161 & NIL & Neg & 62 & Neg & Neg & 62 & $\mathrm{Neg}$ & 62 & 13,545 \\
\hline 162 & NIL & Neg & Neg & Neg & Neg & 74 & Neg & 74 & 4514 \\
\hline 163 & NIL & Neg & 62 & Neg & Neg & 62 & $\mathrm{Neg}$ & 62 & 11,894 \\
\hline 164 & NIL & Neg & Neg & Neg & Neg & Neg & Neg & Neg & ND \\
\hline 165 & NIL & 59 & Neg & Neg & \multicolumn{2}{|c|}{ PCR inhibition } & & & \\
\hline 166 & NIL & Neg & Neg & Neg & Neg & Neg & $\mathrm{Neg}$ & Neg & ND \\
\hline 167 & NIL & 39 & Neg & Other HR & 39 & Neg & 39 & Neg & 52,831 \\
\hline 168 & NIL & Neg & Neg & Neg & Neg & Neg & Neg & Neg & ND \\
\hline 169 & NIL & Neg & Neg & Neg & Neg & Neg & $\mathrm{Neg}$ & Neg & ND \\
\hline 170 & NIL & 66 & Neg & Other HR & 66 & Neg & 66 & Neg & 54,943 \\
\hline 171 & NIL & Neg & Neg & Neg & Neg & Neg & Neg & Neg & ND \\
\hline 172 & NIL & Neg & Neg & Neg & Neg & Neg & $\mathrm{Neg}$ & Neg & ND \\
\hline
\end{tabular}


Table 1 Results of Pap smear, cobas HPV Test, Roche Linear Array HPV Genotyping Test, and Nanopore sequencing (Continued)

\begin{tabular}{|c|c|c|c|c|c|c|c|c|c|}
\hline \multirow[t]{2}{*}{ Patient } & \multirow[t]{2}{*}{ Pap smear } & \multicolumn{2}{|c|}{ Roche Linear Array } & \multirow[t]{2}{*}{ Cobas HPV } & \multicolumn{2}{|c|}{ Nanopore (PGMY) } & \multicolumn{2}{|c|}{ Nanopore (MGP) } & \multirow{2}{*}{$\begin{array}{l}\text { Total } \\
\text { HPV } \\
\text { reads }\end{array}$} \\
\hline & & $\mathrm{HR}$ & Non-HR & & $\mathrm{HR}$ & Non-HR & $\mathrm{HR}$ & Non-HR & \\
\hline 173 & NIL & Neg & Neg & Neg & Neg & Neg & Neg & Neg & ND \\
\hline 174 & NIL & 66 & Neg & Other HR & 66 & Neg & 66 & Neg & 57,791 \\
\hline 175 & NIL & Neg & 54 & Neg & Neg & 54 & Neg & 54 & 23,583 \\
\hline 176 & NIL & Neg & Neg & Neg & \multicolumn{2}{|c|}{ PCR inhibition } & & & \\
\hline 177 & NIL & 16 & 62 & 16 & Neg & 53,62 & 16 & 62 & 28,181 \\
\hline 178 & NIL & Neg & Neg & Neg & Neg & Neg & Neg & Neg & 206 \\
\hline 179 & NIL & Neg & Neg & Neg & Neg & Neg & Neg & Neg & ND \\
\hline 180 & NIL & Neg & Neg & Neg & Neg & Neg & Neg & Neg & ND \\
\hline 181 & NIL & 51,66 & Neg & Other HR & $51,66,68$ & Neg & $51,66,68$ & Neg & 6952 \\
\hline 182 & NIL & $16,51,58$ & 61 & Other HR & 58 & 61 & Neg & 61 & 5737 \\
\hline 183 & NIL & Neg & Neg & Neg & Neg & Neg & Neg & Neg & ND \\
\hline 184 & NIL & 58 & Neg & Other HR & 58 & Neg & 58 & Neg & 43,034 \\
\hline 185 & NIL & 58 & 70,89 & Other HR & 58 & 70,89 & 58 & 89 & 33,842 \\
\hline 186 & ND & Neg & Neg & Neg & Neg & Neg & Neg & Neg & 414 \\
\hline 187 & ND & Neg & Neg & Neg & Neg & Neg & Neg & Neg & ND \\
\hline 188 & ND & 16 & Neg & 16 & 16 & Neg & 16 & Neg & 96,549 \\
\hline 189 & ND & Neg & Neg & Neg & Neg & Neg & Neg & Neg & ND \\
\hline 190 & ND & Neg & Neg & Neg & Neg & Neg & Neg & Neg & ND \\
\hline 191 & ND & 56 & Neg & Other HR & 56 & Neg & 56 & Neg & 18,782 \\
\hline 192 & ND & 51 & Neg & Other HR & 51 & Neg & 51 & Neg & 6020 \\
\hline 193 & ND & Neg & 62 & Neg & Neg & 62 & Neg & 62 & 20,373 \\
\hline 194 & ND & Neg & Neg & Neg & Neg & Neg & Neg & Neg & ND \\
\hline 195 & ND & 52,59 & Neg & Other HR & 52,59 & Neg & 59 & Neg & 11,926 \\
\hline 196 & ND & 59 & Neg & Other HR & 59 & Neg & 59 & Neg & 24,045 \\
\hline 197 & ND & 52,59 & 54,70 & Other HR & 52,59 & 70 & 52,59 & 70,90 & 46,523 \\
\hline 198 & ND & 56,66 & $53,61,84$ & Other HR & 66 & $32,53,61,84$ & 56 & $32,53,61,84$ & 62,600 \\
\hline 199 & ND & Neg & 62 & Neg & Neg & Neg & Neg & Neg & ND \\
\hline 200 & ND & Neg & $53,54,81,83$ & Neg & Neg & $53,54,83$ & Neg & $53,81,83$ & 32,868 \\
\hline 201 & ND & Neg & Neg & Neg & \multicolumn{2}{|c|}{ PCR inhibition } & & & \\
\hline
\end{tabular}

AGUS Atypical glandular cells of undetermined significance, ASCH Atypical squamous cells - cannot exclude HSIL, ASCUS Atypical squamous cells of undetermined significance, HR High-risk, HSIL High-grade squamous intraepithelial lesion, LSIL Low-grade squamous intraepithelial lesion, ND Pap smear/ MinION sequencing not done, Neg Negative, NIL normal cytology

against HPV reference genomes using minimap2 (Galaxy version $2.17+$ galaxy0), and consensus sequences were built from BAM files using Unipro UGENE (version 1.29.0) for determining their percentage of identity to reference genomes.

\section{Results}

As HPV 66 is categorized as 'other high-risk' by cobas HPV Test, all calculations were based on this grouping, albeit HPV 66 was found as a single infection in cancers with extreme rarity and re-classified as possible carcinogen (Group 2B) by IARC Monographs Working Group [6].
The results are summarized in Table 1 . PCR was successful for 191 specimens (191/201, 95.02\%), with 10 specimens $(10 / 201,4.98 \%)$ lacking $\beta$-globin band and therefore regarded as inappropriate for further analysis. Seventy-six specimens $(76 / 201,37.81 \%)$ were negative for both PGMY and MGP PCRs, and 115 (115/201, $57.21 \%$ ) were positive for either of the two. PCR-positive specimens were sequenced on 10 MinION flow cells with 145-890 active pores, generating 31,748-525,880 HPV reads in first $2 \mathrm{~h}$ (Table 4). For the 115 specimens sequenced, 19 were negative (7-522 reads, 113 in average) and 96 were positive (45-96,549 reads, 20,158 in average) for HPV. Taken together, there were $95 \mathrm{HPV}$ - 
Table 2 Primer sequences

\begin{tabular}{|c|c|c|}
\hline Primer & $5^{\prime}$ to $3^{\prime}$ sequence & References \\
\hline \multicolumn{3}{|l|}{ PGMY PCR } \\
\hline PGMY11-A & GCA CAG GGA CAT AAC AAT GG & [13] \\
\hline PGMY11-B & GCG CAG GGC CAC AAT AAT GG & \\
\hline PGMY11-C & GCA CAG GGA CAT AAT AAT GG & \\
\hline PGMY11-D & GCC CAG GGC CAC AAC AAT GG & \\
\hline PGMY11-E & GCT CAG GGT TTA AAC AAT GG & \\
\hline PGMY09-F & CGT CCC AAA GGA AAC TGA TC & \\
\hline PGMY09-G & CGA CCT AAA GGA AAC TGA TC & \\
\hline PGMY09-H & CGT CCA AAA GGA AAC TGA TC & \\
\hline PGMY09-I & G CCA AGG GGA AAC TGA TC & \\
\hline PGMY09-J & CGT CCC AAA GGA TAC TGA TC & \\
\hline PGMY09-K & CGT CCA AGG GGA TAC TGA TC & \\
\hline PGMY09-L & CGA CCT AAA GGG AAT TGA TC & \\
\hline PGMY09-M & CGA CCT AGT GGA AAT TGA TC & \\
\hline PGMY09-N & CGA CCA AGG GGA TAT TGA TC & \\
\hline PGMY09-P & G CCC AAC GGA AAC TGA TC & \\
\hline PGMY09-Q & CGA CCC AAG GGA AAC TGG TC & \\
\hline PGMY09-R & CGT CCT AAA GGA AAC TGG TC & \\
\hline HMB01 & GCG ACC CAA TGC AAA TTG GT & \\
\hline Human $\beta$-globin forward & GAAGAGCCAAGGACAGGTAC & [15] \\
\hline Human $\beta$-globin reverse & GGAAAATAGACCAATAGGCAG & \\
\hline \multicolumn{3}{|l|}{ MGP PCR } \\
\hline MGPA & ACGTTGGATGTTGTTACTGTGGTGGATACTAC & [16] \\
\hline MGPB & ACGTTGGATGTTTGTTACCGTTGTTGATACTAC & \\
\hline MGPC & ACGTTGGATGTTTGTTACTAAGGTAGATACCACTC & \\
\hline MGPD & ACGTTGGATGTTTGTTACTGTTGTGGATACAAC & \\
\hline MGP31 & ACGTTGGATGTTTGTTACTATGGTAGATACCACAC & \\
\hline MGPG & ACGTTGGATGGAAAAATAAACTGTAAATCATATTCCT & \\
\hline MGPH & ACGTTGGATGGAAAAATAAATTGTAAATCATACTC & \\
\hline MGPI & ACGTTGGATGGAAATATAAATTGTAAATCAAATTC & \\
\hline MGPJ & ACGTTGGATGGAAAAATAAACTGTAAATCATATTC & \\
\hline MGP18 & ACGTTGGATGGAAAAATAAACTGCAAATCATATTC & \\
\hline
\end{tabular}

negative (95/201, 47.26\%) and 96 HPV-positive (96/201, 47.76\%) specimens by Nanopore workflow.

Table 5 shows concordance of Nanopore workflow with cobas HPV Test and LA, which was based on the 37 HPV types detectable by LA. For cobas HPV Test, our workflow achieved 93.19, 93.19 and $81.94 \%$ for perfect, total and positive agreement, respectively, with Cohen's kappa of 0.85 . For LA, Nanopore achieved a perfect agreement of $83.77 \%$ for both high-risk and nonhigh risk HPVs. For high-risk types, total and positive agreement were 96.86 and $91.78 \%$, respectively, with Cohen's kappa of 0.93 . For non-high risk types, total and positive agreement were 93.19 and $77.59 \%$, respectively, with Cohen's kappa of 0.83 .

Table 6 shows per-type concordance of Nanopore and LA. A total of 13 high-risk and 19 non-high risk HPV types were evaluated. Positive agreement for HPV 16 $(n=8)$ and $18(n=1)$ were 87.5 and $100 \%$, respectively. Positive agreement was $75-100 \%$ for high-risk HPV 31, $33,35,39,51,52,56,58,59$ and 66 , and $20 \%$ for HPV $68(n=5)$. For non-high risk HPVs, positive agreement was $37.5-100 \%$ for HPV 6, 11, 40, 42, 53, 54, 55, 61, 62, $70,72,73,81,82,83,84$ and 89 . There were 2 non-high risk types with $0 \%$ positive agreement (HPV 26 and 71). 
Table 3 Master mix constituents and PCR conditions PGMY PCR

\begin{tabular}{|c|c|c|}
\hline \multicolumn{2}{|l|}{ Reagent } & Volume/ $\mu \mathrm{L}$ \\
\hline \multicolumn{2}{|c|}{ 10X PCR buffer II (Applied Biosystems) } & 5 \\
\hline \multicolumn{2}{|c|}{25 mM MgCl 2 (Applied Biosystems) } & 3 \\
\hline \multicolumn{2}{|c|}{ PGMY primer mix $(10 \mu M)$} & 1 \\
\hline \multicolumn{2}{|c|}{ Human $\beta$-globin primer mix (5 $\mu \mathrm{M})$} & 1 \\
\hline \multicolumn{2}{|c|}{10 mM dNTPs (Roche) } & 1 \\
\hline \multicolumn{2}{|l|}{$5 \mathrm{M}$ betaine (Sigma) } & 10 \\
\hline \multicolumn{2}{|c|}{ AmpliTaq Gold DNA Polymerase (Applied Biosystems) } & 0.25 \\
\hline \multicolumn{2}{|c|}{ Molecular grade water (Sigma) } & 23.75 \\
\hline \multicolumn{2}{|l|}{ DNA } & 5 \\
\hline \multicolumn{3}{|l|}{ PCR conditions } \\
\hline Temperature $/{ }^{\circ} \mathrm{C}$ & Time & No. of cycles \\
\hline 95 & $9 \min$ & 1 \\
\hline 95 & $1 \mathrm{~min}$ & 40 (50\% ramp) \\
\hline 55 & $1 \mathrm{~min}$ & \\
\hline 72 & $1 \mathrm{~min}$ & \\
\hline 72 & $5 \mathrm{~min}$ & 1 \\
\hline \multirow[t]{2}{*}{15} & Hold & / \\
\hline & IGP PCR & \\
\hline
\end{tabular}

Master mix constituents (for single reaction)

\begin{tabular}{|c|c|c|}
\hline \multicolumn{2}{|l|}{ Reagent } & Volume $/ \mu \mathrm{L}$ \\
\hline \multicolumn{2}{|c|}{ 10X PCR buffer II (Applied Biosystems) } & 2.5 \\
\hline \multicolumn{2}{|c|}{$25 \mathrm{mM} \mathrm{MgCl} 2$ (Applied Biosystems) } & 1.5 \\
\hline \multicolumn{2}{|c|}{ MGP primer mix $(10 \mu \mathrm{M})$} & 0.5 \\
\hline \multicolumn{2}{|c|}{10 mM dNTPs (Roche) } & 0.5 \\
\hline \multicolumn{2}{|c|}{ AmpliTaq Gold DNA Polymerase (Applied Biosystems) } & 0.1 \\
\hline \multicolumn{2}{|c|}{ Molecular grade water (Sigma) } & 14.9 \\
\hline \multicolumn{2}{|l|}{ DNA } & 5 \\
\hline \multicolumn{3}{|l|}{ PCR conditions } \\
\hline \multicolumn{2}{|l|}{ Temperature $/{ }^{\circ} \mathrm{C}$} & No. of cycles \\
\hline 95 & $10 \mathrm{~min}$ & 1 \\
\hline 95 & $30 \mathrm{~s}$ & 5 \\
\hline 42 & $30 \mathrm{~s}$ & \\
\hline 72 & $30 \mathrm{~s}$ & \\
\hline 95 & $30 \mathrm{~s}$ & 45 \\
\hline 64 & $30 \mathrm{~s}$ & \\
\hline 72 & $30 \mathrm{~s}$ & \\
\hline 72 & $5 \mathrm{~min}$ & 1 \\
\hline 15 & Hold & / \\
\hline
\end{tabular}

HPV $26(n=1)$ was only detected by Nanopore workflow, whereas HPV $71(n=2)$ was only detected by LA.

Table 7 reveals the percentage of identity of Nanopore consensus sequences to HPV reference genomes. In
Table 4 Details of Nanopore sequencing runs

\begin{tabular}{llll}
\hline Run & No. of active pores & Elapsed sequencing time & No. of HPV reads \\
\hline 1 & 611 & 2h 11 min & 60,976 \\
2 & 458 & 1 h 59 min & 246,521 \\
3 & 690 & 2h 1 min & 279,520 \\
4 & 467 & 2h 5 min & 111,885 \\
5 & 462 & 2h 5 min & 31,748 \\
6 & 247 & 2h 3 min & 113,521 \\
7 & 330 & 2h 5 min & 111,702 \\
8 & 753 & 2h 1 min & 478,711 \\
9 & 145 & 1h 59 min & 207,094 \\
10 & 890 & 1h 59 min & 525,880
\end{tabular}

general, Nanopore consensus sequences showed an average identity of $98 \%$ to the best matches, with an average difference of $15 \%$ from second BLAST hits.

Table 8 summarizes HPV status of each cytology grading. For high-grade and low-grade squamous intraepithelial lesion (HSIL and LSIL), nearly all specimens were positive for high-risk HPV (HSIL: 4/4, 100\%; LSIL: $16 / 18,88.89 \%)$. For atypical squamous/ glandular cells, about half of the specimens were positive for high-risk HPV (by LA: 19/41, 46.34\%; by Nanopore: 18/41, $43.90 \%)$. For cases without observable abnormalities, $22.12 \%(25 / 113)$ and $21.24 \%(24 / 113)$ were positive for high-risk HPV by LA and Nanopore, respectively.

\section{Discussion}

Hong Kong has been one of the Asian regions with the lowest incidence and mortality rate of CC [16]. This might be attributable to the territory-wide cervical screening program implemented by Department of Health since 2004. The program is well-organized, which involves public education, regular cervical smear and follow-up service for eligible women, and a quality assurance mechanism on key components of the program [17]. Cytology is the mainstay of primary screening, and high-risk HPV testing may be performed for triage to colposcopy.

Cytology and HPV testing have their own value for $\mathrm{CC}$ screening. High quality cytology has high specificity for $\mathrm{CC}$, but with lower sensitivity ranging from $50 \%$ suggested by cross-sectional studies to $75 \%$ estimated longitudinally [18]. For HPV testing, the sensitivity was reported to be about $10 \%$ higher than cytology, yet with lower specificity [18]. Complementary use of both tests could enhance the sensitivity approaching $100 \%$ with high specificity (92.5\%) [19]. In fact, this combined approach has been adopted by several European countries and may become the future trend of primary CC screening in developed countries. 
Table 5 Agreement between cobas HPV Test, Roche Linear Array HPV Genotyping Test (LA) and Nanopore

\begin{tabular}{|c|c|c|c|c|c|c|c|c|}
\hline & & & \multicolumn{2}{|c|}{ Nanopore } & \multirow{2}{*}{$\begin{array}{l}\text { Perfect } \\
\text { agreement }\end{array}$} & \multirow{2}{*}{$\begin{array}{l}\text { Total } \\
\text { agreement }\end{array}$} & \multirow{2}{*}{$\begin{array}{l}\text { Positive } \\
\text { agreement }\end{array}$} & \multirow{2}{*}{$\begin{array}{l}\text { Cohen's } \\
\text { k }\end{array}$} \\
\hline & & & + & - & & & & \\
\hline \multirow[t]{2}{*}{ cobas HPV Test } & + & & 59 & 2 & $93.19 \%$ & $93.19 \%$ & $81.94 \%$ & 0.85 \\
\hline & - & & 11 & 119 & & & & \\
\hline \multirow[t]{4}{*}{ LA } & HR & + & 67 & 4 & $83.77 \%$ & $96.86 \%$ & $91.78 \%$ & 0.93 \\
\hline & & - & 2 & 118 & & & & \\
\hline & Non-HR & + & 45 & 10 & & $93.19 \%$ & $77.59 \%$ & 0.83 \\
\hline & & - & 3 & 133 & & & & \\
\hline
\end{tabular}

Table 6 Per HPV type positive agreement between Roche Linear Array Genotyping Test (LA) and Nanopore

\begin{tabular}{|c|c|c|c|c|c|c|c|}
\hline \multirow[t]{2}{*}{ HPV Genotypes } & & \multicolumn{5}{|l|}{ Number of specimens } & \multirow{2}{*}{$\begin{array}{l}\text { Positive } \\
\text { agreement }\end{array}$} \\
\hline & & Nanopore-/LA-/LA- & Nanopore +/LA- & Nanopore-/LA+ & Nanopore+/LA+ & Total & \\
\hline \multirow[t]{13}{*}{ High-risk } & 16 & 183 & 0 & 1 & 7 & 191 & $87.5 \%$ \\
\hline & 18 & 190 & 0 & 0 & 1 & 191 & $100 \%$ \\
\hline & 31 & 188 & 0 & 0 & 3 & 191 & $100 \%$ \\
\hline & 33 & 189 & 0 & 0 & 2 & 191 & $100 \%$ \\
\hline & 35 & 190 & 0 & 0 & 1 & 191 & $100 \%$ \\
\hline & 39 & 185 & 0 & 1 & 5 & 191 & $83.33 \%$ \\
\hline & 51 & 182 & 0 & 1 & 8 & 191 & $88.89 \%$ \\
\hline & 52 & 165 & 3 & 2 & 21 & 191 & $80.77 \%$ \\
\hline & 56 & 185 & 0 & 0 & 6 & 191 & $100 \%$ \\
\hline & 58 & 184 & 0 & 0 & 7 & 191 & $100 \%$ \\
\hline & 59 & 179 & 2 & 1 & 9 & 191 & $75 \%$ \\
\hline & 66 & 182 & 1 & 0 & 8 & 191 & $88.89 \%$ \\
\hline & 68 & 186 & 2 & 2 & 1 & 191 & $20 \%$ \\
\hline \multirow[t]{19}{*}{ Non-high risk } & 6 & 190 & 0 & 0 & 1 & 191 & $100 \%$ \\
\hline & 11 & 189 & 0 & 0 & 2 & 191 & $100 \%$ \\
\hline & 26 & 190 & 1 & 0 & 0 & 191 & $0 \%$ \\
\hline & 40 & 187 & 2 & 0 & 2 & 191 & $50 \%$ \\
\hline & 42 & 186 & 2 & 1 & 2 & 191 & $40 \%$ \\
\hline & 53 & 181 & 3 & 0 & 7 & 191 & $70 \%$ \\
\hline & 54 & 181 & 0 & 4 & 6 & 191 & $60 \%$ \\
\hline & 55 & 186 & 0 & 0 & 5 & 191 & $100 \%$ \\
\hline & 61 & 186 & 0 & 0 & 5 & 191 & $100 \%$ \\
\hline & 62 & 174 & 2 & 2 & 13 & 191 & $76.47 \%$ \\
\hline & 70 & 188 & 0 & 0 & 3 & 191 & $100 \%$ \\
\hline & 71 & 189 & 0 & 2 & 0 & 191 & $0 \%$ \\
\hline & 72 & 190 & 0 & 0 & 1 & 191 & $100 \%$ \\
\hline & 73 & 190 & 0 & 0 & 1 & 191 & $100 \%$ \\
\hline & 81 & 182 & 0 & 1 & 8 & 191 & $88.89 \%$ \\
\hline & 82 & 189 & 0 & 0 & 2 & 191 & $100 \%$ \\
\hline & 83 & 189 & 0 & 0 & 2 & 191 & $100 \%$ \\
\hline & 84 & 183 & 0 & 5 & 3 & 191 & $37.5 \%$ \\
\hline & 89 & 188 & 0 & 0 & 3 & 191 & $100 \%$ \\
\hline
\end{tabular}


Table 7 Percentage of identity of Nanopore consensus sequences to HPV reference genomes

\begin{tabular}{|c|c|c|c|c|c|c|}
\hline \multirow[t]{2}{*}{ Patient } & \multirow{2}{*}{$\begin{array}{l}\text { Nanopore } \\
\text { results }\end{array}$} & \multicolumn{2}{|c|}{ Best BLAST hit } & \multicolumn{2}{|c|}{ Second BLAST hit } & \multirow[t]{2}{*}{ Difference } \\
\hline & & HPV type & $\%$ identity & HPV type & $\%$ identity & \\
\hline \multirow[t]{2}{*}{2} & 59 & 59 & $99 \%$ & 18 & $77 \%$ & $22 \%$ \\
\hline & ${ }^{a} 90$ & 90 & $97 \%$ & 106 & $84 \%$ & $15 \%$ \\
\hline \multirow[t]{2}{*}{3} & 52 & 52 & $99 \%$ & 58 & $80 \%$ & $19 \%$ \\
\hline & 55 & 55 & $100 \%$ & 44 & $93 \%$ & $7 \%$ \\
\hline \multirow[t]{4}{*}{5} & 31 & 31 & $98 \%$ & 35 & $80 \%$ & $18 \%$ \\
\hline & 33 & 33 & $99 \%$ & 58 & $86 \%$ & $13 \%$ \\
\hline & ${ }^{a} 52$ & 52 & $99 \%$ & 58 & $80 \%$ & $19 \%$ \\
\hline & ${ }^{\mathrm{a}} 90$ & 90 & $97 \%$ & 106 & $85 \%$ & $12 \%$ \\
\hline 7 & 31 & 31 & $95 \%$ & 35 & $79 \%$ & $16 \%$ \\
\hline 9 & 81 & 81 & $99 \%$ & 62 & $85 \%$ & $14 \%$ \\
\hline 10 & 18 & 18 & $99 \%$ & 45 & $85 \%$ & $14 \%$ \\
\hline \multirow[t]{5}{*}{13} & ${ }^{\mathrm{a}} 44$ & 44 & $99 \%$ & 55 & $92 \%$ & $7 \%$ \\
\hline & 52 & 52 & $99 \%$ & 58 & $80 \%$ & $19 \%$ \\
\hline & 53 & 53 & $99 \%$ & 30 & $85 \%$ & $14 \%$ \\
\hline & ${ }^{\mathrm{a}} 74$ & 74 & $99 \%$ & 55 & $83 \%$ & $16 \%$ \\
\hline & ${ }^{\mathrm{a}} 90$ & 90 & $97 \%$ & 106 & $85 \%$ & $12 \%$ \\
\hline \multirow[t]{2}{*}{16} & 52 & 52 & $99 \%$ & 58 & $81 \%$ & $18 \%$ \\
\hline & 81 & 81 & $99 \%$ & 62 & $85 \%$ & $14 \%$ \\
\hline \multirow[t]{2}{*}{17} & 52 & 52 & $99 \%$ & 58 & $80 \%$ & $19 \%$ \\
\hline & 54 & 54 & $99 \%$ & 45 & $74 \%$ & $25 \%$ \\
\hline \multirow[t]{3}{*}{18} & 11 & 11 & $99 \%$ & 6 & $87 \%$ & $12 \%$ \\
\hline & 52 & 52 & $99 \%$ & 58 & $80 \%$ & $19 \%$ \\
\hline & 59 & 59 & $99 \%$ & 18 & $77 \%$ & $22 \%$ \\
\hline \multirow[t]{4}{*}{23} & 39 & 39 & $99 \%$ & 70 & $81 \%$ & $18 \%$ \\
\hline & 61 & 61 & $99 \%$ & mEV06c12b & $83 \%$ & $16 \%$ \\
\hline & 72 & 72 & $92 \%$ & mEV06c12b & $89 \%$ & $3 \%$ \\
\hline & a 87 & 87 & $98 \%$ & 86 & $85 \%$ & $13 \%$ \\
\hline 24 & 66 & 66 & $98 \%$ & 56 & $84 \%$ & $14 \%$ \\
\hline 25 & 61 & 61 & $99 \%$ & mEV06c12b & $83 \%$ & $16 \%$ \\
\hline 26 & ${ }^{\mathrm{a}} 90$ & 90 & $97 \%$ & 106 & $85 \%$ & $12 \%$ \\
\hline \multirow[t]{2}{*}{27} & 52 & 52 & $99 \%$ & 58 & $80 \%$ & $19 \%$ \\
\hline & ${ }^{a} 87$ & 87 & $98 \%$ & 86 & $84 \%$ & $14 \%$ \\
\hline 28 & 62 & 62 & $99 \%$ & 81 & $84 \%$ & $15 \%$ \\
\hline 30 & 35 & 35 & $98 \%$ & 31 & $80 \%$ & $18 \%$ \\
\hline 32 & 52 & 52 & $99 \%$ & 58 & $81 \%$ & $18 \%$ \\
\hline 34 & 51 & 51 & $99 \%$ & 82 & $85 \%$ & $14 \%$ \\
\hline 35 & ${ }^{a} 74$ & 74 & $99 \%$ & 55 & $84 \%$ & $15 \%$ \\
\hline 37 & 51 & 51 & $99 \%$ & 82 & $85 \%$ & $14 \%$ \\
\hline \multirow[t]{3}{*}{38} & 40 & 40 & $99 \%$ & 7 & $88 \%$ & $11 \%$ \\
\hline & 55 & 55 & $99 \%$ & 44 & $93 \%$ & $6 \%$ \\
\hline & 83 & 83 & $99 \%$ & 102 & $84 \%$ & $15 \%$ \\
\hline \multirow[t]{2}{*}{40} & ${ }^{a} 52$ & 52 & $99 \%$ & 58 & $80 \%$ & $19 \%$ \\
\hline & 53 & 53 & $98 \%$ & 30 & $85 \%$ & $13 \%$ \\
\hline
\end{tabular}


Table 7 Percentage of identity of Nanopore consensus sequences to HPV reference genomes (Continued)

\begin{tabular}{|c|c|c|c|c|c|c|}
\hline \multirow[t]{2}{*}{ Patient } & \multirow{2}{*}{$\begin{array}{l}\text { Nanopore } \\
\text { results }\end{array}$} & \multicolumn{2}{|c|}{ Best BLAST hit } & \multicolumn{2}{|c|}{ Second BLAST hit } & \multirow[t]{2}{*}{ Difference } \\
\hline & & HPV type & $\%$ identity & HPV type & $\%$ identity & \\
\hline & 55 & 55 & $100 \%$ & 44 & $93 \%$ & $7 \%$ \\
\hline & 58 & 58 & $99 \%$ & 33 & $86 \%$ & $13 \%$ \\
\hline & 62 & 62 & $99 \%$ & 81 & $85 \%$ & $14 \%$ \\
\hline & ${ }^{\mathrm{a}} 74$ & 74 & $98 \%$ & 55 & $84 \%$ & $14 \%$ \\
\hline \multirow[t]{3}{*}{41} & 42 & 42 & $98 \%$ & 32 & $83 \%$ & $15 \%$ \\
\hline & 52 & 52 & $100 \%$ & 58 & $81 \%$ & $19 \%$ \\
\hline & 73 & 73 & $99 \%$ & 34 & $85 \%$ & $14 \%$ \\
\hline 43 & 16 & 16 & $100 \%$ & 35 & $78 \%$ & $22 \%$ \\
\hline 44 & 16 & 16 & $99 \%$ & 35 & $78 \%$ & $21 \%$ \\
\hline 45 & 59 & 59 & $99 \%$ & 18 & $76 \%$ & $23 \%$ \\
\hline \multirow[t]{2}{*}{46} & 31 & 31 & $98 \%$ & 35 & $80 \%$ & $18 \%$ \\
\hline & 58 & 58 & $99 \%$ & 33 & $86 \%$ & $13 \%$ \\
\hline \multirow[t]{4}{*}{47} & 52 & 52 & $98 \%$ & 58 & $80 \%$ & $18 \%$ \\
\hline & 68 & 68 & $93 \%$ & 39 & $81 \%$ & $12 \%$ \\
\hline & 84 & 84 & $98 \%$ & 87 & $84 \%$ & $14 \%$ \\
\hline & ${ }^{a} 90$ & 90 & $97 \%$ & 106 & $85 \%$ & $12 \%$ \\
\hline \multirow[t]{3}{*}{48} & ${ }^{a} 44$ & 44 & $99 \%$ & 55 & $93 \%$ & $6 \%$ \\
\hline & 66 & 66 & $98 \%$ & 56 & $84 \%$ & $14 \%$ \\
\hline & 84 & 84 & $99 \%$ & 87 & $84 \%$ & $15 \%$ \\
\hline 49 & 52 & 52 & $99 \%$ & 58 & $80 \%$ & $19 \%$ \\
\hline \multirow[t]{2}{*}{50} & 40 & 40 & $98 \%$ & 7 & $87 \%$ & $11 \%$ \\
\hline & 53 & 53 & $98 \%$ & 30 & $85 \%$ & $13 \%$ \\
\hline \multirow[t]{4}{*}{51} & 11 & 11 & $100 \%$ & 6 & $87 \%$ & $13 \%$ \\
\hline & ${ }^{\mathrm{a}} 43$ & 43 & $95 \%$ & 45 & $77 \%$ & $18 \%$ \\
\hline & 52 & 52 & $99 \%$ & 58 & $80 \%$ & $19 \%$ \\
\hline & 81 & 81 & $99 \%$ & 62 & $84 \%$ & $15 \%$ \\
\hline 52 & 66 & 66 & $98 \%$ & 56 & $83 \%$ & $15 \%$ \\
\hline \multirow[t]{3}{*}{53} & ${ }^{a} 43$ & 43 & $95 \%$ & 45 & $78 \%$ & $17 \%$ \\
\hline & 51 & 51 & $99 \%$ & 82 & $84 \%$ & $15 \%$ \\
\hline & ${ }^{\mathrm{a}} 90$ & 90 & $97 \%$ & 106 & $85 \%$ & $12 \%$ \\
\hline \multirow[t]{7}{*}{54} & 16 & 16 & $100 \%$ & 35 & $78 \%$ & $22 \%$ \\
\hline & ${ }^{a} 40$ & 40 & $93 \%$ & 7 & $85 \%$ & $8 \%$ \\
\hline & 51 & 51 & $99 \%$ & 82 & $84 \%$ & $15 \%$ \\
\hline & 54 & 54 & $99 \%$ & 45 & $73 \%$ & $26 \%$ \\
\hline & 56 & 56 & $90 \%$ & 66 & $76 \%$ & $14 \%$ \\
\hline & 62 & 62 & $99 \%$ & 81 & $84 \%$ & $15 \%$ \\
\hline & 81 & 81 & $99 \%$ & 62 & $85 \%$ & $14 \%$ \\
\hline \multirow[t]{2}{*}{55} & 53 & 53 & $99 \%$ & 56 & $79 \%$ & $20 \%$ \\
\hline & 56 & 56 & $99 \%$ & 66 & $84 \%$ & $15 \%$ \\
\hline \multirow[t]{4}{*}{57} & 54 & 54 & $87 \%$ & 31 & $74 \%$ & $13 \%$ \\
\hline & 55 & 55 & $100 \%$ & 44 & $93 \%$ & $7 \%$ \\
\hline & 66 & 66 & $98 \%$ & 56 & $84 \%$ & $14 \%$ \\
\hline & 81 & 81 & $99 \%$ & 62 & $84 \%$ & $15 \%$ \\
\hline
\end{tabular}


Table 7 Percentage of identity of Nanopore consensus sequences to HPV reference genomes (Continued)

\begin{tabular}{|c|c|c|c|c|c|c|}
\hline \multirow[t]{2}{*}{ Patient } & \multirow{2}{*}{$\begin{array}{l}\text { Nanopore } \\
\text { results }\end{array}$} & \multicolumn{2}{|c|}{ Best BLAST hit } & \multicolumn{2}{|c|}{ Second BLAST hit } & \multirow[t]{2}{*}{ Difference } \\
\hline & & HPV type & $\%$ identity & HPV type & $\%$ identity & \\
\hline & ${ }^{\mathrm{a}} 90$ & 90 & $97 \%$ & 106 & $85 \%$ & $12 \%$ \\
\hline \multirow[t]{3}{*}{58} & ${ }^{\mathrm{a}} 42$ & 42 & $99 \%$ & 32 & $84 \%$ & $15 \%$ \\
\hline & 52 & 52 & $98 \%$ & 58 & $80 \%$ & $18 \%$ \\
\hline & ${ }^{\mathrm{a}} 90$ & 90 & $97 \%$ & 106 & $85 \%$ & $12 \%$ \\
\hline 59 & 59 & 59 & $99 \%$ & 18 & $77 \%$ & $22 \%$ \\
\hline \multirow[t]{2}{*}{60} & 59 & 59 & $99 \%$ & 18 & $76 \%$ & $23 \%$ \\
\hline & 89 & 89 & $99 \%$ & 81 & $78 \%$ & $21 \%$ \\
\hline \multirow[t]{3}{*}{61} & ${ }^{a} 43$ & 43 & $96 \%$ & 45 & $79 \%$ & $17 \%$ \\
\hline & 56 & 56 & $97 \%$ & 66 & $83 \%$ & $14 \%$ \\
\hline & 82 & 82 & $99 \%$ & 51 & $84 \%$ & $15 \%$ \\
\hline 62 & 52 & 52 & $99 \%$ & 58 & $80 \%$ & $19 \%$ \\
\hline \multirow[t]{3}{*}{63} & 33 & 33 & $99 \%$ & 58 & $86 \%$ & $13 \%$ \\
\hline & ${ }^{\mathrm{a}} 44$ & 44 & $99 \%$ & 55 & $93 \%$ & $6 \%$ \\
\hline & 51 & 51 & $99 \%$ & 82 & $83 \%$ & $16 \%$ \\
\hline 64 & 51 & 51 & $99 \%$ & 82 & $84 \%$ & $15 \%$ \\
\hline 65 & 16 & 16 & $100 \%$ & 35 & $78 \%$ & $22 \%$ \\
\hline 68 & ${ }^{a} 59$ & 59 & $99 \%$ & 18 & $77 \%$ & $22 \%$ \\
\hline 69 & a 87 & 87 & $99 \%$ & 86 & $86 \%$ & $13 \%$ \\
\hline \multirow[t]{3}{*}{74} & ${ }^{a} 52$ & 52 & $99 \%$ & 58 & $81 \%$ & $18 \%$ \\
\hline & 58 & 58 & $99 \%$ & 33 & $86 \%$ & $13 \%$ \\
\hline & ${ }^{\mathrm{a}} 62$ & 62 & $99 \%$ & 81 & $85 \%$ & $14 \%$ \\
\hline 75 & 58 & 58 & $99 \%$ & 33 & $85 \%$ & $14 \%$ \\
\hline 78 & ${ }^{\mathrm{a}} 90$ & 90 & $97 \%$ & 106 & $85 \%$ & $12 \%$ \\
\hline \multirow[t]{3}{*}{79} & ${ }^{\mathrm{a}} 44$ & 44 & $99 \%$ & 55 & $92 \%$ & $7 \%$ \\
\hline & 56 & 56 & $96 \%$ & 66 & $84 \%$ & $12 \%$ \\
\hline & 70 & 70 & $99 \%$ & 39 & $81 \%$ & $18 \%$ \\
\hline 81 & ${ }^{\mathrm{a}} 74$ & 74 & $93 \%$ & 55 & $81 \%$ & $12 \%$ \\
\hline 82 & 42 & 42 & $95 \%$ & 32 & $83 \%$ & $12 \%$ \\
\hline 83 & ${ }^{\mathrm{a}} 74$ & 74 & $97 \%$ & 55 & $83 \%$ & $14 \%$ \\
\hline 85 & 82 & 82 & $99 \%$ & 51 & $84 \%$ & $15 \%$ \\
\hline 86 & 62 & 62 & $99 \%$ & 81 & $85 \%$ & $14 \%$ \\
\hline \multirow[t]{2}{*}{91} & 52 & 52 & $99 \%$ & 58 & $80 \%$ & $19 \%$ \\
\hline & ${ }^{\mathrm{a}} 90$ & 90 & $97 \%$ & 106 & $84 \%$ & $13 \%$ \\
\hline \multirow[t]{2}{*}{92} & ${ }^{\mathrm{a}} 42$ & 42 & $93 \%$ & 32 & $78 \%$ & $15 \%$ \\
\hline & 68 & 68 & $92 \%$ & 39 & $80 \%$ & $12 \%$ \\
\hline 96 & 52 & 52 & $99 \%$ & 58 & $80 \%$ & $19 \%$ \\
\hline 100 & 52 & 52 & $99 \%$ & 58 & $80 \%$ & $19 \%$ \\
\hline 104 & 62 & 62 & $98 \%$ & 81 & $85 \%$ & $13 \%$ \\
\hline \multirow[t]{4}{*}{107} & ${ }^{\mathrm{a}} 44$ & 44 & $99 \%$ & 55 & $93 \%$ & $6 \%$ \\
\hline & 52 & 52 & $99 \%$ & 58 & $81 \%$ & $18 \%$ \\
\hline & ${ }^{a} 53$ & 53 & $100 \%$ & 30 & $86 \%$ & $14 \%$ \\
\hline & 62 & 62 & $99 \%$ & 81 & $85 \%$ & $14 \%$ \\
\hline 112 & 16 & 16 & $98 \%$ & 58 & $78 \%$ & $20 \%$ \\
\hline
\end{tabular}


Table 7 Percentage of identity of Nanopore consensus sequences to HPV reference genomes (Continued)

\begin{tabular}{|c|c|c|c|c|c|c|}
\hline \multirow[t]{2}{*}{ Patient } & \multirow{2}{*}{$\begin{array}{l}\text { Nanopore } \\
\text { results }\end{array}$} & \multicolumn{2}{|c|}{ Best BLAST hit } & \multicolumn{2}{|c|}{ Second BLAST hit } & \multirow[t]{2}{*}{ Difference } \\
\hline & & HPV type & $\%$ identity & HPV type & $\%$ identity & \\
\hline & 52 & 52 & $99 \%$ & 58 & $81 \%$ & $18 \%$ \\
\hline \multirow[t]{5}{*}{114} & ${ }^{\mathrm{a}} 26$ & 26 & $100 \%$ & 69 & $83 \%$ & $17 \%$ \\
\hline & 55 & 55 & $100 \%$ & 44 & $93 \%$ & $7 \%$ \\
\hline & $a_{59}$ & 59 & $99 \%$ & 18 & $77 \%$ & $22 \%$ \\
\hline & ${ }^{\mathrm{a}} 62$ & 62 & $99 \%$ & 81 & $85 \%$ & $14 \%$ \\
\hline & 89 & 89 & $99 \%$ & 81 & $77 \%$ & $22 \%$ \\
\hline 115 & ${ }^{\mathrm{a}} 74$ & 74 & $95 \%$ & 55 & $83 \%$ & $12 \%$ \\
\hline \multirow[t]{2}{*}{118} & 6 & 6 & $99 \%$ & 11 & $87 \%$ & $12 \%$ \\
\hline & 62 & 62 & $99 \%$ & 81 & $84 \%$ & $15 \%$ \\
\hline 124 & 81 & 81 & $99 \%$ & 62 & $85 \%$ & $14 \%$ \\
\hline 125 & ${ }^{a} 87$ & 87 & $98 \%$ & 86 & $85 \%$ & $13 \%$ \\
\hline 126 & ${ }^{\mathrm{a}} 90$ & 90 & $97 \%$ & 106 & $85 \%$ & $12 \%$ \\
\hline 138 & 39 & 39 & $99 \%$ & 68 & $81 \%$ & $18 \%$ \\
\hline \multirow[t]{4}{*}{143} & ${ }^{a} 40$ & 40 & $99 \%$ & 7 & $88 \%$ & $11 \%$ \\
\hline & ${ }^{a} 74$ & 74 & $98 \%$ & 55 & $84 \%$ & $14 \%$ \\
\hline & 81 & 81 & $99 \%$ & 62 & $84 \%$ & $15 \%$ \\
\hline & ${ }^{a} 87$ & 87 & $97 \%$ & 86 & $84 \%$ & $13 \%$ \\
\hline 148 & 59 & 59 & $99 \%$ & 18 & $77 \%$ & $22 \%$ \\
\hline 152 & 62 & 62 & $98 \%$ & 81 & $85 \%$ & $13 \%$ \\
\hline \multirow[t]{2}{*}{156} & 52 & 52 & $99 \%$ & 58 & $81 \%$ & $18 \%$ \\
\hline & 54 & 54 & $95 \%$ & 6 & $74 \%$ & $21 \%$ \\
\hline \multirow[t]{3}{*}{157} & 39 & 39 & $94 \%$ & 70 & $81 \%$ & $13 \%$ \\
\hline & 53 & 53 & $96 \%$ & 30 & $84 \%$ & $12 \%$ \\
\hline & 61 & 61 & $99 \%$ & mEV06c12b & $83 \%$ & $16 \%$ \\
\hline 161 & 62 & 62 & $98 \%$ & 81 & $83 \%$ & $15 \%$ \\
\hline 162 & ${ }^{a} 74$ & 74 & $94 \%$ & 55 & $85 \%$ & $9 \%$ \\
\hline 163 & 62 & 62 & $99 \%$ & 81 & $85 \%$ & $14 \%$ \\
\hline 167 & 39 & 39 & $99 \%$ & 70 & $81 \%$ & $18 \%$ \\
\hline 170 & 66 & 66 & $98 \%$ & 56 & $83 \%$ & $15 \%$ \\
\hline 174 & 66 & 66 & $98 \%$ & 56 & $83 \%$ & $15 \%$ \\
\hline 175 & 54 & 54 & $99 \%$ & 45 & $73 \%$ & $26 \%$ \\
\hline \multirow[t]{3}{*}{177} & 16 & 16 & $99 \%$ & 35 & $80 \%$ & $19 \%$ \\
\hline & ${ }^{a} 53$ & 53 & $99 \%$ & 30 & $84 \%$ & $15 \%$ \\
\hline & 62 & 62 & $99 \%$ & 81 & $85 \%$ & $14 \%$ \\
\hline \multirow[t]{3}{*}{181} & 51 & 51 & $99 \%$ & 82 & $85 \%$ & $14 \%$ \\
\hline & 66 & 66 & $98 \%$ & 56 & $83 \%$ & $15 \%$ \\
\hline & ${ }^{a} 68$ & 68 & $98 \%$ & 39 & $81 \%$ & $17 \%$ \\
\hline \multirow[t]{2}{*}{182} & 58 & 58 & $98 \%$ & 33 & $87 \%$ & $11 \%$ \\
\hline & 61 & 61 & $100 \%$ & mEV06c12b & $83 \%$ & $17 \%$ \\
\hline 184 & 58 & 58 & $99 \%$ & 33 & $85 \%$ & $14 \%$ \\
\hline \multirow[t]{3}{*}{185} & 58 & 58 & $99 \%$ & 33 & $85 \%$ & $14 \%$ \\
\hline & 70 & 70 & $99 \%$ & 39 & $81 \%$ & $18 \%$ \\
\hline & 89 & 89 & $99 \%$ & 81 & $78 \%$ & $21 \%$ \\
\hline
\end{tabular}


Table 7 Percentage of identity of Nanopore consensus sequences to HPV reference genomes (Continued)

\begin{tabular}{|c|c|c|c|c|c|c|}
\hline \multirow[t]{2}{*}{ Patient } & \multirow{2}{*}{$\begin{array}{l}\text { Nanopore } \\
\text { results }\end{array}$} & \multicolumn{2}{|c|}{ Best BLAST hit } & \multicolumn{2}{|c|}{ Second BLAST hit } & \multirow[t]{2}{*}{ Difference } \\
\hline & & HPV type & $\%$ identity & HPV type & $\%$ identity & \\
\hline 188 & 16 & 16 & $100 \%$ & 35 & $78 \%$ & $22 \%$ \\
\hline 191 & 56 & 56 & $99 \%$ & 66 & $83 \%$ & $16 \%$ \\
\hline 192 & 51 & 51 & $98 \%$ & 82 & $84 \%$ & $14 \%$ \\
\hline 193 & 62 & 62 & $99 \%$ & 81 & $85 \%$ & $14 \%$ \\
\hline \multirow[t]{2}{*}{195} & 52 & 52 & $99 \%$ & 58 & $81 \%$ & $18 \%$ \\
\hline & 59 & 59 & $99 \%$ & 18 & $76 \%$ & $23 \%$ \\
\hline 196 & 59 & 59 & $99 \%$ & 18 & $77 \%$ & $22 \%$ \\
\hline \multirow[t]{4}{*}{197} & 52 & 52 & $100 \%$ & 58 & $81 \%$ & $19 \%$ \\
\hline & 59 & 59 & $99 \%$ & 18 & $76 \%$ & $23 \%$ \\
\hline & 70 & 70 & $99 \%$ & 39 & $81 \%$ & $18 \%$ \\
\hline & ${ }^{\mathrm{a}} 90$ & 90 & $97 \%$ & 106 & $85 \%$ & $12 \%$ \\
\hline \multirow[t]{6}{*}{198} & ${ }^{a} 32$ & 32 & $99 \%$ & 42 & $84 \%$ & $15 \%$ \\
\hline & 53 & 53 & $99 \%$ & 30 & $86 \%$ & $13 \%$ \\
\hline & 56 & 56 & $99 \%$ & 66 & $84 \%$ & $15 \%$ \\
\hline & 61 & 61 & $100 \%$ & mEV06c12b & $83 \%$ & $17 \%$ \\
\hline & 66 & 66 & $98 \%$ & 56 & $83 \%$ & $15 \%$ \\
\hline & 84 & 84 & $99 \%$ & 87 & $84 \%$ & $15 \%$ \\
\hline \multirow[t]{4}{*}{200} & 53 & 53 & $98 \%$ & 30 & $85 \%$ & $13 \%$ \\
\hline & 54 & 54 & $99 \%$ & 45 & $74 \%$ & $25 \%$ \\
\hline & 81 & 81 & $99 \%$ & 62 & $84 \%$ & $15 \%$ \\
\hline & 83 & 83 & $95 \%$ & 102 & $82 \%$ & $13 \%$ \\
\hline \multicolumn{3}{|c|}{ Average $\%$ identity of the best hit } & $98 \%$ & \multicolumn{2}{|c|}{ Average difference } & $15 \%$ \\
\hline
\end{tabular}

${ }^{a}$ HPV types not detected by LA

Table 8 Results of Pap smear, LA and Nanopore workflow. The calculations were based 176 quality control-valid specimens with Pap smear results available

\begin{tabular}{llll}
\hline Pap smear interpretation & HPV status & \multicolumn{2}{l}{ No. of specimens } \\
\cline { 3 - 4 } & & LA & Nanopore \\
\hline HSIL $(n=4)$ & HR/ HR + non-HR & 4 & 4 \\
& Non-HR only & 0 & 0 \\
& Negative & 0 & 0 \\
LSIL/ LSIL + ASCH $(n=18)$ & HR/ HR + non-HR & 16 & 16 \\
& Non-HR only & 1 & 1 \\
& Negative & 1 & 1 \\
AGUS/ ASCH/ ASCUS ( $n=41)$ & HR/ HR + non-HR & 19 & 18 \\
& Non-HR only & 3 & 6 \\
& Negative & 19 & 17 \\
& HR/ HR + non-HR & 25 & 24 \\
& Non-HR only & 18 & 18 \\
& Negative & 70 & 71 \\
\hline
\end{tabular}

Compared with HPV assays in the market, HPV genotyping by NGS offers a broader detection spectrum which, despite minimal benefit of non-high risk HPV information for $\mathrm{CC}$ screening, may provide important etiologic clues for other HPV-associated infections and a more complete picture of HPV epidemiology. For the latter, Nanopore identified more HPV types per sample (Fig. 1) and 5 extra HPV types (HPV 43, 44, 74, 87 and $90, n=34$ ) not detectable by LA (Fig. 2), with an unexpected high incidence of HPV $90(n=12)$ which was reported in North America and Belgium but not in Hong Kong [20, 21]. Another advantage offered by NGS is its potential utility for simultaneous characterization of cervicovaginal microbiome, with its possible role in dysplasia and carcinogenesis revealed by accumulating research evidence [22-25]. These merits may facilitate a multifaceted approach for evaluation of woman health in near feature.

In general, Nanopore had substantial agreement with cobas HPV Test and LA. Compared with cobas HPV Test, Nanopore appeared to be more sensitive for HPV $52(n=7)$ and $59(n=4)$, with $81.82 \%(9 / 11)$ of these discrepant results matched with LA. Compared with LA, 


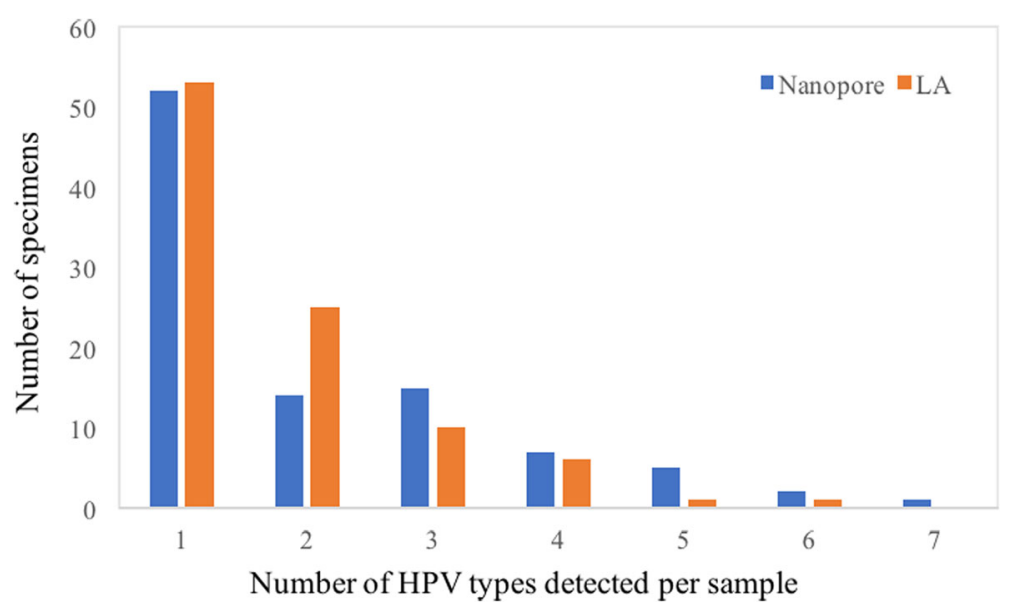

Fig. 1 Number of HPV types detected per sample by Nanopore workflow and LA

concordance for high-risk HPV was higher than nonhigh risk types. Among the 37 discrepant results, 22 were false negatives by Nanopore and 15 were not detected by LA.

For the false negatives by Nanopore, more than half $(12 / 22,54.55 \%)$ were mixed infections, and similar finding was reported by other research groups using HPV consensus primers for NGS-based genotyping [10, 11]. Other possible causes of false negatives included (1) low viral load, as evident by Specimen 182, from which HPV 16 was missed by both Nanopore and cobas HPV Test; (2) substantial difference in DNA input $(50 \mu \mathrm{L}$ for LA versus $5 \mu \mathrm{L}$ for PGMY/ MGP PCR), as well as (3) lower sensitivity due to reduced magnesium chloride concentration of PGMY PCR (from $4 \mathrm{mM}$ to $1.5 \mathrm{mM}$ ), which was fine-tuned for minimal non-specific amplification.

For the 15 HPV types missed by LA, the average identity of Nanopore consensus sequences was $98.27 \%$ with an average difference of $16 \%$ from second BLAST hits (Table 7). As distinct HPV types generally have more than $10 \%$ difference in L1 sequence [26, 27], it appeared that the discrepant positive calls were less likely caused by high sequencing error rate of Nanopore. More specifically, 5 of these positive calls were identified solely by MGP PCR (5/15, 33.33\%), 5 detected by PGMY PCR only $(5 / 15,33.33 \%)$, and 5 by both PCRs $(5 / 15,33.33 \%)$. These revealed differential sensitivities of PGMY and

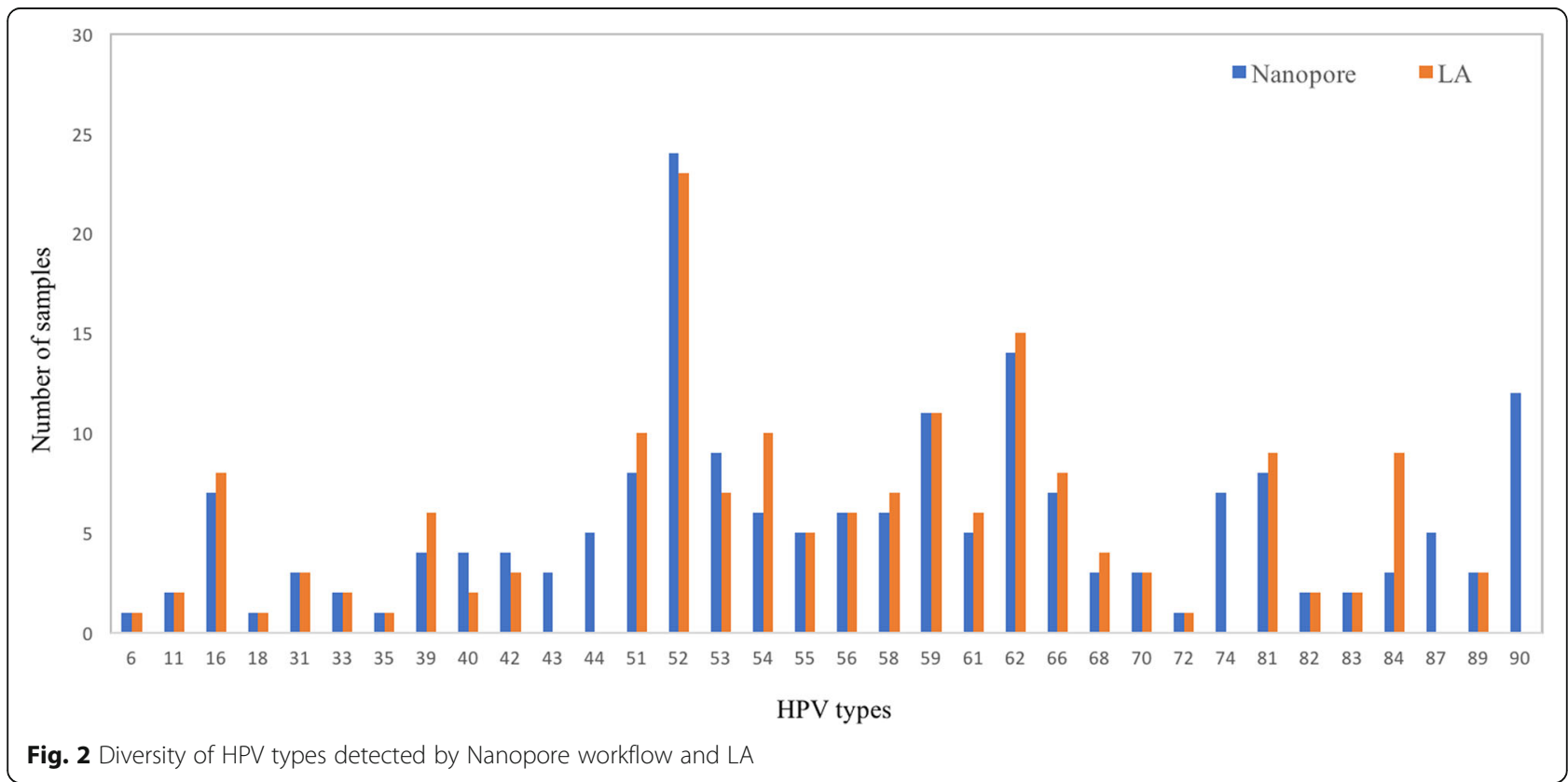


Table 9 Comparison of estimated reagent cost of Nanopore workflow (24-plex) and randomly-selected prices of HPV genotyping referral service in Hong Kong

\begin{tabular}{|c|c|c|}
\hline \multicolumn{3}{|l|}{ This study } \\
\hline Procedure & Number of specimens & Cost \\
\hline $\begin{array}{l}\text { DNA extraction } \\
\text { and PCRs }\end{array}$ & $\begin{array}{l}201 \text { patients }+20 \\
\text { controls }=221\end{array}$ & $\begin{array}{l}\text { USD } 20.02 \times 221 \\
\text { reactions }=\text { USD } 4424.42\end{array}$ \\
\hline $\begin{array}{l}\text { Nanopore } \\
\text { sequencing }\end{array}$ & $\begin{array}{l}115 \text { patients } / 24=\text { at } \\
\text { least } 5 \text { runs } \\
N=120 \text { for } 1 \text { positive } \\
\text { control per run }\end{array}$ & $\begin{array}{l}\text { USD } 1155.94 \times 5 \text { runs }= \\
\text { USD } 5779.70\end{array}$ \\
\hline \multicolumn{2}{|c|}{ Cost per patient specimen } & $\begin{array}{l}(4424.42+5779.70) / 201= \\
\text { USD } 50.77\end{array}$ \\
\hline \multicolumn{3}{|c|}{ Referral service (transportation cost not included) } \\
\hline \multicolumn{2}{|l|}{ Lab A } & USD 77.19 \\
\hline \multicolumn{2}{|l|}{ Lab B } & USD 124.79 \\
\hline \multicolumn{2}{|l|}{ Lab C } & USD 101.63 \\
\hline \multicolumn{2}{|l|}{ Lab D } & USD 120.93 \\
\hline \multicolumn{2}{|l|}{ Average } & USD 106.14 \\
\hline
\end{tabular}

MGP PCR primers, which might complement with each other and enhance overall performance of the Nanopore assay. On the other hand, Nanopore sequencing might improve the resolution of genotyping, which might not be attained by line blot method due to crosshybridization of certain probes. For instance, Nanopore identified HPV 52 in Specimen 5, 40 and 74, which could not be confirmed by LA due to crosshybridization with HPV 33 and 58, respectively. Another example was Specimen 125, which was HPV 84-positive by LA and HPV 87-positive by Nanopore. From literature, Artaza-Irigaray and colleagues reported crosshybridization between these 2 HPV types by LA, with 11.5\% of HPV 84-positive cervical specimens by LA were actually HPV 87-positive by NGS [28].

The Nanopore method and LA revealed very similar high-risk HPV positivity in each cytology grading. The goal of combined cytology-HPV testing approach is to enhance cost effectiveness of CC screening. While minimizing unnecessary referral for colposcopy, HPV genotyping may identify high-risk individuals before observable cytological abnormalities, for instance, the 4 HPV 16-positive patients without abnormal cytology findings in this study. This may facilitate an early detection approach for cancer prevention.

Our study had several limitations. First, the sample size of certain HPV types, for example, HPV $18(n=1)$, was less satisfactory for evaluating type-specific performance. Second, as residual DNA was used after routine testing, DNA input for PGMY and MGP PCRs was constrained which might lower the sensitivity. In addition, as flow cells with suboptimal number of active pores were used, sequencing time and depth might be further improved if new flow cells were used.

\section{Conclusions}

We developed a Nanopore workflow for HPV genotyping, with performance comparable to or better than 2 reference methods in the market. Our method was economical, with a reagent cost of about USD 50.77 per patient specimen for 24-plex runs, which was competitive when compared to an average price of USD 106.14 (from 4 randomly-selected laboratories) for HPV genotyping referral service in our region (Table 9). The protocol was also straightforward with reasonable turnaround time of about $12 \mathrm{~h}$ from samples to answers. The small size and portability of MinION sequencers may well suit remote or resource-limited laboratories with constraints in space. Future prospective study with larger sample size is warranted to further evaluate test performance and streamline the protocol. As LA was discontinued in Hong Kong, the Nanopore workflow described here may provide an economical option for broad-range HPV genotyping.

\section{Abbreviations \\ CC: Cervical cancer; HPV: Human papillomavirus; HSIL: High-grade squamous intraepithelial lesion; IARC: International Agency for Research on Cancer (IARC); LA: Roche Linear Array HPV Genotyping Test; LSIL: Low-grade squamous intraepithelial lesion; NGS: Next-generation sequencing; Pap smear: Papanicolaou smear; PCR: Polymerase chain reaction; WHO: World Health Organization}

\section{Acknowledgements}

We thank the colleagues of Department of Pathology, Hong Kong Sanatorium \& Hospital for their dedicated and professional work on routine laboratory diagnostics.

\section{Authors' contributions}

BSFT, TLC and WSC conceived and designed the study. BSFT, ESKM, MKMC, TLC, CPL, CHA, MYT, MKN, SML and WSC were involved in data collection and analysis. WSC wrote the first draft. All authors critically reviewed and approved the manuscript.

\section{Funding}

Not applicable.

\section{Availability of data and materials}

The datasets used and/or analyzed during the current study are available from the corresponding author on reasonable request.

\section{Ethics approval and consent to participate}

This study was approved by Research Ethics Committee (REC) of Hong Kong Sanatorium \& Hospital under the reference number RC-2019-18. No patientidentifying data was collected throughout the whole study.

Consent for publication

Not applicable.

Competing interests

The authors declare that they have no competing interests. 
Received: 20 January 2020 Accepted: 28 April 2020

Published online: 06 May 2020

\section{References}

1. zur Hausen $\mathrm{H}$. Papillomaviruses in the causation of human cancers - a brief historical account. Virology. 2009;384:260-5.

2. Meisels A, Fortin R. Condylomatous lesions of the cervix and vagina. I Cytologic patterns Acta Cytol. 1976;20:505-9.

3. Purola E, Savia E. Cytology of gynecologic condyloma acuminatum. Acta Cytol. 1977;21:26-31.

4. Muñoz N, Bosch FX, de Sanjosé S, Herrero R, Castellsagué X, Shah KV, et al. Epidemiologic classification of human papillomavirus types associated with cervical cancer. N Engl J Med. 2003;348:518-27.

5. IARC Working Group on the Evaluation of Carcinogenic Risks to Humans. Human papillomaviruses. IARC Monogr Eval Carinog Risks Hum. 2007:90:1-636.

6. Schiffman M, Clifford G, Buonaguro FM. Classification of weakly carcinogenic human papillomavirus types: addressing the limits of epidemiology at the borderline. Infect Agent Cancer. 2009;4:8.

7. Chrysostomou AC, Stylianou DC, Constantinidou A, Kostrikis LG. Cervical cancer screening programs in Europe: the transition towards HPV vaccination and population-based HPV testing. Viruses. 2018;10:729.

8. Petry KU, Barth C, Wasem J, Neumann A. A model to evaluate the costs and clinical effectiveness of human papilloma virus screening compared with annual Papanicolaou cytology in Germany. Eur J Obstet Gynecol Reprod Biol. 2017;212:132-9.

9. Pan American Health Organization. Section 2: Summary of commercially available HPV tests. https://www.paho.org/hq/dmdocuments/2016/manualVPH-English-02.pdf (2016). Accessed 3 Jan 2020.

10. Nilyanimit P, Chansaenroj J, Poomipak W, Praianantathavorn K, Payungporn S, Poovorawan Y. Comparison of four human papillomavirus genotyping methods: next-generation sequencing, INNO-LiPA, electrochemical DNA Chip, and nested-PCR. Ann Lab Med. 2018;38:139-46.

11. Nowak RG, Ambulos NP, Schumaker LM, Mathias TJ, White RA, Troyer J, et al. Genotyping of high-risk anal human papillomavirus (HPV): ion torrentnext generation sequencing vs. linear array. Virol J. 2017;14:112.

12. Wagner S, Roberson D, Boland J, Yeager M, Cullen M, Mirabello L, et al. Development of the TypeSeq assay for detection of 51 human papillomavirus genotypes by next-generation sequencing. J Clin Microbiol. 2019;57:e01794-18.

13. Gravitt PE, Peyton CL, Alessi TQ, Wheeler CM, Coutlée F, Hildesheim A, et al. Improved amplification of genital human papillomaviruses. J Clin Microbiol. 2000:38:357-61.

14. Söderlund-Strand A, Carlson J, Dillner J. Modified general primer PCR system for sensitive detection of multiple types of oncogenic human papillomavirus. J Clin Microbiol. 2009;47:541-6.

15. Marín M, Garcia-Lechuz JM, Alonso P, Villanueva M, Alcalá L, Gimeno M, et al. Role of universal 16S rRNA gene PCR and sequencing in diagnosis of prosthetic joint infection. J Clin Microbiol. 2012;50:583-9.

16. Department of Health, the Government of the Hong Kong Special Administrative Region. Evidence for organized screening programme. https://www.cervicalscreening.gov.hk/english/about/abt_evidence.html (2013). Accessed 15 Jan 2020.

17. Department of Health, the Government of the Hong Kong Special Administrative Region. About Cervical Screening Programme. https://wmw.cervicalscreening.gov. hk/english/about/about.html (2013). Accessed 15 Jan 2020

18. World Health Organization: Cervical cancer screening in developing countries. Report of a WHO consultation. https:/apps.who.int/iris/bitstream/ handle/10665/42544/9241545720.pdf;jsessionid=2599A27FFB141B755D015 B645FB889D9? sequence=1 (2002). Accessed 15 Jan 2020.

19. Mayrand MH, Duarte-Franco E, Rodrigues I, Walter SD, Hanley J, Ferenczy A, et al. Human papillomavirus DNA versus Papanicolaou screening tests for cervical cancer. N Engl J Med. 2007;357:1579-88.

20. Quiroga-Garza G, Zhou H, Mody DR, Schwartz MR, Ge Y. Unexpected high prevalence of HPV 90 infection in an underserved population: is it really a low-risk genotype? Arch Pathol Lab Med. 2013;137:1569-73.

21. Schmitt M, Depuydt C, Benoy I, Bogers J, Antoine J, Arbyn M, et al. Prevalence and viral load of 51 genital human papillomavirus types and three subtypes. Int J Cancer. 2013;132:2395-403.

22. Mitra A, Maclntyre DA, Lee YS, Smith A, Marchesi JR, Lehne B, et al. Cervical intraepithelial neoplasia disease progression is associated with increased vaginal microbiome diversity. Sci Rep. 2015;5:16865.
23. Oh HY, Kim BS, Seo SS, Kong JS, Lee JK, Park SY, et al. The association of uterine cervical microbiota with an increased risk for cervical intraepithelial neoplasia in Korea. Clin Microbiol Infect. 2015;21:674 e1-9.

24. Klein C, Gonzalez D, Samwel K, Kahesa C, Mwaiselage J, Aluthge N, et al. Relationship between the cervical microbiome, HIV status, and precancerous lesions. MBio. 2019;10:e02785-18.

25. Nené NR, Reisel D, Leimbach A, Franchi D, Jones A, Evans I, et al. Association between the cervicovaginal microbiome, BRCA1 mutation status, and risk of ovarian cancer: a case-control study. Lancet Oncol. 2019; 20:1171-82.

26. King AJ, Sonsma JA, Vriend HJ, van der Sande MA, Feltkamp MC, Boot HJ, et al. Genetic diversity in the major capsid L1 protein of HPV-16 and HPV-18 in the Netherlands. PLoS One. 2016;11:e0152782.

27. Burk RD, Harari A, Chen Z. Human papillomavirus genome variants. Virology. 2013:445:232-43.

28. Artaza-Irigaray C, Flores-Miramontes MG, Olszewski D, Vallejo-Ruiz V, LimónToledo LP, Sánchez-Roque C, et al. Cross-hybridization between HPV genotypes in the linear Array genotyping test confirmed by next-generation sequencing. Diagn Pathol. 2019;14:31.

\section{Publisher's Note}

Springer Nature remains neutral with regard to jurisdictional claims in published maps and institutional affiliations.
Ready to submit your research? Choose BMC and benefit from:

- fast, convenient online submission

- thorough peer review by experienced researchers in your field

- rapid publication on acceptance

- support for research data, including large and complex data types

- gold Open Access which fosters wider collaboration and increased citations

- maximum visibility for your research: over $100 \mathrm{M}$ website views per year

At BMC, research is always in progress.

Learn more biomedcentral.com/submissions 